Relations industrielles

Industrial Relations

\title{
Des loisirs : pour quoi faire? Par Jean Fourastié, Tournai, Casterman, 1970, 143 pp.
}

André Roy

Volume 28, numéro 1, 1973

URI : https://id.erudit.org/iderudit/028386ar

DOI : https://doi.org/10.7202/028386ar

Aller au sommaire du numéro

Éditeur(s)

Département des relations industrielles de l'Université Laval

ISSN

0034-379X (imprimé)

1703-8138 (numérique)

Découvrir la revue

Citer ce compte rendu

Roy, A. (1973). Compte rendu de [Des loisirs : pour quoi faire? Par Jean

Fourastié, Tournai, Casterman, 1970, 143 pp.] Relations industrielles / Industrial

Relations, 28(1), 223-225. https://doi.org/10.7202/028386ar

Tous droits réservés @ C Département des relations industrielles de l'Universite Laval, 1973
Ce document est protégé par la loi sur le droit d'auteur. L’utilisation des services d'Érudit (y compris la reproduction) est assujettie à sa politique d'utilisation que vous pouvez consulter en ligne.

https://apropos.erudit.org/fr/usagers/politique-dutilisation/ 
del is then tested on data for several manufacturing industries. Variable construction is discussed, and results of estimation and tests are reported. For example, this wage equation can be interpreted as a Phillips curve to which three 《bargaining variables》 have been added. These variables are : a form of net-value productivity per worker, the supply price of labor to the union, and the gross layoff rate to reflect general conditions in the economy as a whole and specific conditions to the industry. When estimates of the wage equation were compared to estimates of a simple Phillips curve without these bargaining variables, the equation explained the quarterly movement of average hourly earnings in the test industries better than the simple Phillips curve.

There is no doubt that de Menil's Bargaining : Monopoly versus Union Power provides a much needed theoretical basis for examining the influence of product market forces on wages and for analysing union - non-union relative wages. However, some criticisms are needed. First, the author's review of bargaining theories is far from being complete. Only slight reference is made to the «utility functions approach 》 which includes more than the works of Zeuthen and Harsanyi. The major omissions are probably the contributions of J. Pen, Allan M. Cartter, Pao Lu Cheng, Bevars Dupre Mabry. On the other hand, no reference at all is made to what could be called the «behavioralist approach 》 i.e. to the works of Ann Douglas, Carl M. Stevens and R.E. Walton and R.B. McKersie.

Secondly, the approach taken by de Menil, presents the usual shortcomings of similar studies : It is an oversimplification of the reality that exists behind collective agreements. This is so because it deals solely with the determination of wages and ignores the total relationships between the parties which collective bargaining seeks to establish ; and also because, like other branches of the theory of games, it makes the simplistic assumption that each party in the bargaining process has full knowledge of the objective payoffs associated with every possible outcome and of the utility functions of his opponent.
Much remains to be said about bargaining power and the variables which determine the outcome of collective bargaining.

Jean BOIVIN

Des loisirs : pourquoi faire?, par Jean Fourastié, Tournai, Casterman, 1970, 143 pp.

Jean Fourastié est un économiste bien connu, surtout par ses études sur le devenir des sociétés contemporaines. Il suffit de penser à quelques-uns de ses ouvrages, en particulier Le Grand Espoir du XXe siècle, La civilisation de 1975, Les quarante mille heures, où il a tenté de percer le secret de l'avenir. Il a pratiqué la prospective avant qu'il ne soit encore question de cette discipline encore toute jeune.

Dans ce court ouvrage, l'auteur essaie de prévoir dans quelle mesure diminuera, au cours des prochains vingt ans, le temps consacré au travail dans la vie de l'homme et à quoi sera occupé un temps de loisir de plus en plus long.

En effet, cette époque n'est-elle pas déjà à nos portes? Quelques phénomènes idéologiques actuels ne permettent-ils pas de le constater? Par exemple, il ne fait pas de doute que dans certains milieux, face au spectre de la pollution, de la contamination plus ou moins marquée de l'environnement, qu'il s'agisse de l'air, de l'eau, de la terre, des esprits, que certains disent pessimistes, estiment qu'il faudra assez peu de temps avant que la terre devienne proprement inhabitable. D'autres croient que, au rythme de la production actuelle, il ne s'écoulera pas non plus beaucoup de générations avant que les ressources de la planète ne viennent à s'épuiser, d'où la nécessité de tendre dès maintenant à l'élaboration d'une société dans laquelle on se préoccupera davantage de la qualité de la vie que de l'accroissement sans frein des biens de consommation. De plus, on peut se demander si le développement effrené du secteur tertiaire depuis la fin de la guerre dans les pays industrialisés ne nous a pas fait entrer sans que nous nous en rendions bien compte dans ce nouvel âge. On ne peut non plus ignorer que le nombre s'accroît sans cesse de ceux qui, face à l'ampleur d'un chô- 
mage toujours récurrent, estiment que, au lieu de se torturer les méninges pour créer des emplois, il vaudrait mieux jeter aux orties les vieux concepts économiques pour s'orienter dans une voie toute nouvelle où l'on viserait avant tout non pas tant à créer des emplois nouveaux qu'à assurer aux hommes les nécessités de la vie. Ce sont là, face aux réalités actuelles, des courants d'idées assez profonds pour que nous ne pensions plus à les considérer comme des chimères et des utopies, mais qui s'imposent à notre réflexion.

Même si l'on reste attaché aux principes de l'économie capitaliste tels qu'ils sont enseignés depuis une couple de siècles, il est impossible de ne pas voir dans certaines préoccupations actuelles un signe du temps qui change. Par exemple, l'O.C.D.E. a tenu, au début de l'automne dernier, un congrès pendant lequel furent présentés plusieurs travaux sur l'aménagement de la durée du travail : diminution de la journée ou de la semaine de travail, établissement de la semaine de quatre jours, aménagement d'horaires de travail flexibles, abaissement de l'âge de la retraite, et, ce qui est le plus frappant, prolongement de la période de congés annuels payés. Sur ce dernier sujet, en se fondant sur une prévision de l'accroissement de la productivité d'ici à 1980 , on est arrivé aux conclusions surprenantes suivantes. Selon que la croissance de la productivité serait de 3.2 , de 4.4 ou de 5.2 pour cent par année, en allouant les deux premiers tiers à l'augmentation du niveau de vie général et le dernier tiers à la prolongation des congés annuels payés, on arriverait en 1980, selon le pourcentage de l'accroissement de la productivité, à l'établissement de congés annuels payés variant entre huit et douze semaines par année. On se rend compte, quelles que soient les modalités d'aménagement de la durée du travail, que l'on peut facilement prévoir dans un avenir assez prochain une forte augmentation au temps consacré aux loisirs.

$\mathrm{Vu}$ dans cette perspective, le petit ouvrage de Jean Fourastié revêt une signification singulière et il ne peut être que de très grande actualité. L'auteur parle sans doute pour la France, mais sa portée en dépasse beaucoup les frontières.
Le premier chapitre est consacré à un retour en arrière. L'auteur y rappelle ce qu'étaient les loisirs et le travail d'autrefois. Même si l'homme produisait beaucoup moins qu'aujourd'hui, s'il était exposé à une mort prématurée, voire à la famine, il trouvait quand même le tour de se donner du bon temps, de se distraire et de se récréer. D'ailleurs, comme le travail s'accomplissait avec lenteur, qu'il était pour ainsi dire lié à la vie, libéré en quelque sorte du souci d'efficacité, il lui était loisible de mêler à l'activité laborieuse, «nille récréations, mille petites joies qui faisaient désirer de vivre ».

Fourastié passe ensuite à l'étude du phénomène de la durée du travail. Il s'agit ici encore pour lui d'envisager la question à travers le cheminernent de l'histoire, mais d'une histoire plus près de nous. Il y remarque qu'avec «l'avènement de l'ère scientifique qui a permis peu à peu une augmentation de la consommation, l'amélioration du niveau de vie devint plus que jamais la préoccupation dominante », ce qui n'empêche pas que, dans un peu plus d'un siècle, la durée de la semaine de travail dans l'industrie est passée de 72 heures à 40 heures approximativement. Aussi notet-il que la croissance de la productivité offre à l'homme contemporain trois possibilités: produire plus, travailler moins pour une production constante, travailler moins pour une production moindre. Face à cette situation bien concrète, bien réelle, Fourastié opte pour la voie du compromis: «C'est pourquoi, désormais, écrit-il, il ne s'agit plus autant, du moins dans les pays industrialisés, d'augmenter la production, mais de trouver un compromis entre la double aspiration contradictoire de l'homme : élever son niveau de vie, améliorer son genre de vie; il faut en effet plus ou moins sacrifier l'un pour avoir l'autre ». A ce sujet, considérant les modalités multiples que peut prendre la recherche d'une vie plus civilisée pour l'homme, il aborde la question même des loisirs en analysant les relations entre le travail et les loisirs, montrant la nécessité absolue des loisirs dans un climat où de plus en plus, la fatigue s'installe à demeure, signalant les dangers de bâtir des formes de loisirs qui ne font que changer le mal de place. «Ce dont l'homme a besoin pour se déve- 
lopper, écrit-il, c'est de calme, de nature, de silence, de tranquilité, de vrai sommeil, d'une activité physique avec une alimentation équilibrée $»$, de contacts humains véritablement enrichissants.

Et le regard de l'auteur se porte alors sur demain. Il a fixé une date : 1989 ; mais il se pourrait bien, l'évolution suivant son cours normal, que cette date fut devancée de quelque dix ans. Dans une série de raccourcis saisissants, il essaie de prévoir les traits caractéristiques de la condition humaine à cette époque : accès pour tous à la culture, à l'enseignement, cérébralisation des masses, c'est-à-dire que, dans beaucoup de pays, l'humanité aura atteint le point où l'activité physique tiendra une place moins importante que l'activité intellectuelle et émotive, dépersonnalisation considérable du milieu par suite du poids de contraintes administratives anonymes et interchangeables, prolifération de la réglementation, d'où l'impression d'être constamment victime de l'arbitraire, de l'injustice, causes de sentiments d'insatisfaction et de révolte, uniformité accrue du milieu technique, diversité de besoins toujours croissants que l'homme ne parvient jamais à satisfaire, tendance de plus en plus marquée à la diversification des choix, accroissement de la richesse ou généralisation de la société d'abondance.

Prévisions que tout cela de la part de l'auteur! Il se peut bien que non. Ce n'est peut-être, au fond, qu'observation de ce qui se passe sous les yeux sans qu'il soit possible de s'en rendre bien compte. Déjà, on pressent que les pièces se placent l'une après l'autre sur l'échiquier.

Pour l'homme d'abord, la question fondamentale qui se posera sera de faire la juste part entre le quantitatif et le qualitatif, ce qui fera naître bien des impasses, exigera nombre de décisions audacieuses de la part des individus autant que des pouvoirs publics : lutte contre la frénésie de la vie quotidienne, conflit entre la tendance à consommer et les loisirs, aménagement de la durée du travail, rationnement de l'espace, protection de la nature.

La réponse à la question que pose le titre de ce petit volume: Des loisirs, pour quoi faire?, on peut la découvrir dans ces quelques phrases : «Le loisir n'est pas, n'est plus, un élément secondaire de la réalité humaine. L'emploi du temps libre est la pierre de touche de la personnalité. Dans la mesure où la durée de ce temps libre augmente, par la réduction de la durée des temps contraints, le loisir devient un élément essentiel de la condition humaine ».

Ce petit ouvrage est un livre précieux à l'heure actuelle. Il renferme mille observations qui ne peuvent que susciter des réflexions salutaires, peut-être surtout chez ceux qui restent, aujourd'hui encore les yeux tournés vers des théories économiques et sociales compréhensibles il y a vingt-cinq ans, mais qui apparaissent singulièrement dépassées aujourd'hui.

\section{André ROY}

\section{Readings in Labor Economics and Labor}

Relations, by Richard L. Rowan (ed.), Homewood, Illinois, Richard D. Irwin Inc., 1972, $621 \mathrm{pp}$.

Cet ouvrage regroupe 55 articles dans des domaines aussi vastes que l'histoire des relations de travail, l'économique du travail, la négociation collective et ce que l'éditeur appelle les relations industrielles. C'est un livre qui se veut, par le critère de sélection des articles, complément des textes de base dans le domaine.

L'éditeur a regroupé les articles sous six titres principaux. Examinons-les rapidement. Dans une introduction aux relations de travail et à l'économique du travail, les articles choisis insistent surtout sur l'aspect « ressources humaines 》 des relations industrielles. On y définit la main-d'oeuvre et le chômage, on présente des projections de main-d'oeuvre, on parle des femmes sur le marché du travail, etc. On y retrouve les Gardner, les Rosow, les Bloom, les Northrup, les Perella, etc. On aurait souhaité y voir des articles sur les changements technologiques.

La deuxième partie présente très rapidement l'histoire du mouvement syndical américain. Des articles plus «théoriques» (Dunlop, Taylor) fournissent quelques explications sur le mouvement syndical et précèdent les Commons, Grob, Perlman, Hoxie et Taft dont cha- 